\title{
Duality and semi-group property for backward parabolic Ito equations *
}

\author{
Nikolai Dokuchaev \\ Department of Mathematics and Statistics, Curtin University of Technology, \\ GPO Box U1987, Perth, Western Australia, 6845
}

November 15, 2018

\begin{abstract}
We study existence, uniqueness, semi-group property, and a priori estimates for solutions for backward parabolic Ito equations in domains with boundary. We study also duality between forward and backward equations. The semi-group for backward equations is established in the form of some anti-causality. The novelty is that the semi-group property involves the diffusion term that is a part of the solution.
\end{abstract}

AMS 1991 subject classification: Primary 60J55, 60J60, 60H10. Secondary 34F05, 34G10.

Key words and phrases: parabolic Ito equations, backward SPDEs, regularity, semi-group property, anti-causality

\section{Introduction}

The paper studies backward stochastic partial differential equations (SPDEs) with a Dirichlet boundary condition in a cylinder $D \times[0, T]$ for a region $D \subseteq \mathbf{R}^{n}$ with boundary condition at the terminal time $t=T$. The difference between backward and forward equations is not that important for the deterministic equations since a backward equation can be converted to a forward equation by a time change. However, it cannot be done so easily for stochastic equations, because one looks for a solution adapted to the driving Brownian motion. It is why the backward stochastic differential equations require special consideration. A possible approach is to consider

${ }^{*}$ Random Operators and Stochastic Equations. (2010) 18, 51-72. 
the so-called Bismut backward equations such the diffusion term is not given a priori but needs to be found. These backward SPDEs were widely studied (see, e.g., Yong and Zhou (1999) and references there; non-linear ordinary backward Ito equations was studied in Pardoux and Peng (1990); backward SPDEs was studied also by Dokuchaev (1992), (2003)). Note that there is a duality between linear forward and backward equations. Forward SPDEs were studied in the literature (see, e.g., Alós et al (1999), Bally et al (1994), Chojnowska-Michalik and Goldys (1995), Da Prato and Tubaro (1996), Gyöngy (1998), Krylov (1999), Maslowski (1995), Pardoux (1993), Rozovskii (1990), Walsh (1986), Zhou (1992), Dokuchaev (1995), (2002), (2005), and the bibliography there). Duality between forward and backward equations was studied by Zhou (1992) for domains without boundary and by Dokuchaev (1992) for domains with boundary in some special cases. Backward SPDEs represent analogs of backward parabolic Kolmogorov equations for non-Markov Ito processes, including the case of bounded domains, so they may be used for characterization of distributions of first exit time in non-Markovian setting, as was shown by the author (1992). A different type of backward equations was described in Chapter 5 of Rozovskii (1990).

In the present paper, we study existence, uniqueness, a priori estimates, duality, and semigroup properties for solutions for backward linear parabolic Ito equations in domains. The novelty is that we consider domains with boundaries; the semi-group property involves the diffusion term that is a part of the solution. The proofs for prior estimates are based on duality between forward and backward equations, so the most part of the paper is devoted to establishing this duality. This duality is used also to establish that backward parabolic equations have some causality (more precisely, some anti-causality). This fact can help, for instance, to split time intervals and apply numerical methods via using of auxiliary problems with pathwise constant in time coefficients, or to apply dynamic programming methods for the corresponding control problems.

\section{Definitions}

\subsection{Spaces and classes of functions.}

Assume that we a given an open domain $D \subseteq \mathbf{R}^{n}$ such that either $D=\mathbf{R}^{n}$ or $D$ is bounded with $C^{2}$-smooth boundary $\partial D$. Let $T>0$ be given, and let $Q \triangleq D \times(0, T)$.

We are given a standard complete probability space $(\Omega, \mathcal{F}, \mathbf{P})$ and a right-continuous filtration $\mathcal{F}_{t}$ of complete $\sigma$-algebras of events, $t \geq 0$. We are given also a $N$-dimensional process 
$w(t)=\left(w_{1}(t), \ldots, w_{N}(t)\right)$ with independent components such that it is a Wiener process with respect to $\mathcal{F}_{t}$.

We denote by $\|\cdot\|_{X}$ the norm in a linear normed space $X$, and $(\cdot, \cdot)_{X}$ denote the scalar product in a Hilbert space $X$.

We introduce some spaces of real valued functions.

Let $G \subset \mathbf{R}^{k}$ be an open domain, then $W_{q}^{m}(G)$ denote the Sobolev space of functions that belong to $L_{q}(G)$ with the distributional derivatives up to the $m$ th order, $q \geq 1$.

We denote Euclidean norm in $\mathbf{R}^{k}$ as $|\cdot|$, and $\bar{G}$ denote the closure of a region $G \subset \mathbf{R}^{k}$.

Let $H^{0} \triangleq L_{2}(D)$, and let $H^{1} \triangleq \stackrel{0}{=} W_{2}^{1}(D)$ be the closure in the $W_{2}^{1}(D)$-norm of the set of all smooth functions $u: D \rightarrow \mathbf{R}$ such that $\left.u\right|_{\partial D} \equiv 0$. Let $H^{2}=W_{2}^{2}(D) \cap H^{1}$ be the space equipped with the norm of $W_{2}^{2}(D)$. The spaces $H^{k}$ and $W_{2}^{k}(D)$ are called Sobolev spaces, they are Hilbert spaces, and $H^{k}$ is a closed subspace of $W_{2}^{k}(D), k=0,1,2$.

Let $H^{-1}$ be the dual space to $H^{1}$, with the norm $\|\cdot\|_{H^{-1}}$ such that if $u \in H^{0}$ then $\|u\|_{H^{-1}}$ is the supremum of $(u, v)_{H^{0}}$ over all $v \in H^{1}$ such that $\|v\|_{H^{1}} \leq 1 . H^{-1}$ is a Hilbert space.

We shall write $(u, v)_{H^{0}}$ for $u \in H^{-1}$ and $v \in H^{1}$, meaning the obvious extension of the bilinear form from $u \in H^{0}$ and $v \in H^{1}$.

We denote by $\bar{\ell}_{k}$ the Lebesgue measure in $\mathbf{R}^{k}$, and we denote by $\overline{\mathcal{B}}_{k}$ the $\sigma$-algebra of Lebesgue sets in $\mathbf{R}^{k}$.

We denote by $\overline{\mathcal{P}}$ the completion (with respect to the measure $\bar{\ell}_{1} \times \mathbf{P}$ ) of the $\sigma$-algebra of subsets of $[0, T] \times \Omega$, generated by functions that are progressively measurable with respect to $\mathcal{F}_{t}$.

Let $Q_{s} \triangleq D \times[s, T]$. For $k=-1,0,1,2$, we introduce the spaces

$X^{k}(s, T) \triangleq L^{2}\left([s, T] \times \Omega, \overline{\mathcal{P}}, \bar{\ell}_{1} \times \mathbf{P} ; H^{k}\right), \quad Z_{t}^{k} \triangleq L^{2}\left(\Omega, \mathcal{F}_{t}, \mathbf{P} ; H^{k}\right), \quad \mathcal{C}^{k}(s, T) \triangleq C\left([s, T] ; Z_{T}^{k}\right)$,

Furthermore, introduce the spaces

$$
Y^{k}(s, T) \triangleq X^{k}(s, T) \cap \mathcal{C}^{k-1}(s, T), \quad k \geq 0,
$$

with the norm $\|u\|_{Y^{k}(s, T)} \triangleq\|u\|_{X^{k}(s, T)}+\|u\|_{\mathcal{C}^{k-1}(s, T)}$.

For brevity, we shall use the notations $X^{k} \triangleq X^{k}(0, T), \mathcal{C}^{k} \triangleq \mathcal{C}^{k}(0, T)$, and $Y^{k} \triangleq Y^{k}(0, T)$.

The spaces $X^{k}$ and $Z_{t}^{k}$ are Hilbert spaces.

Further, introduce the spaces

$$
\mathcal{W}_{p}^{k} \triangleq L^{2}\left([0, T] \times \Omega, \overline{\mathcal{P}}, \bar{\ell}_{1} \times \mathbf{P} ; W_{p}^{k}(D)\right), \quad k=0,1, \ldots, \quad 1 \leq p \leq+\infty
$$


The same notation we shall use the space of $n \times n$-dimensional matrix functions. In that case, $\|\cdot\|_{\mathcal{W}_{p}^{k}}$ means the summa of all this norms for all components.

Proposition 2.1 Let $\xi \in X^{0}$, let a sequence $\left\{\xi_{k}\right\}_{k=1}^{+\infty} \subset L^{\infty}\left([0, T] \times \Omega, \ell_{1} \times \mathbf{P} ; C(D)\right)$ be such that all $\xi_{k}(\cdot, t, \omega)$ are progressively measurable with respect to $\mathcal{F}_{t}$, and let $\left\|\xi-\xi_{k}\right\|_{X^{0}} \rightarrow 0$. Let $t \in[0, T]$ and $j \in\{1, \ldots, N\}$ be given. Then the sequence of the integrals $\int_{0}^{t} \xi_{k}(x, s, \omega) d w_{j}(s)$ converges in $Z_{t}^{0}$ as $k \rightarrow \infty$, and its limit depends on $\xi$, but does not depend on $\left\{\xi_{k}\right\}$.

Proof follows from completeness of $X^{0}$ and from the equality

$$
\mathbf{E} \int_{0}^{t}\left\|\xi_{k}(\cdot, s, \omega)-\xi_{m}(\cdot, s, \omega)\right\|_{H^{0}}^{2} d s=\int_{D} d x \mathbf{E}\left(\int_{0}^{t}\left(\xi_{k}(x, s, \omega)-\xi_{m}(x, s, \omega)\right) d w_{j}(s)\right)^{2}
$$

Definition 2.2 Let $\xi \in X^{0}, t \in[0, T], j \in\{1, \ldots, N\}$, then we define $\int_{0}^{t} \xi(x, s, \omega) d w_{j}(s)$ as the limit in $Z_{t}^{0}$ as $k \rightarrow \infty$ of a sequence $\int_{0}^{t} \xi_{k}(x, s, \omega) d w_{j}(s)$, where the sequence $\left\{\xi_{k}\right\}$ is such as in Proposition 2.1.

Sometimes we shall omit $\omega$.

For $t \in[0, T]$, define operators $\delta_{t}: C\left([0, T] ; Z_{T}^{k}\right) \rightarrow Z_{t}^{k}$ such that $\delta_{t} u=u(\cdot, t)$, where $k=-1,0,1$.

\section{Review of existence results for forward equations}

Let $s \in[0, T), \varphi \in X^{-1}, h_{i} \in X^{0}$, and $\Phi \in Z_{s}^{0}$. Consider the problem

$$
\begin{aligned}
& d_{t} u=(\mathcal{A} u+\varphi) d t+\sum_{i=1}^{N}\left[B_{i} u+h_{i}\right] d w_{i}(t), \quad t \geq s, \\
& \left.u\right|_{t=s}=\Phi,\left.\quad u(x, t, \omega)\right|_{x \in \partial D}=0 .
\end{aligned}
$$

Here $u=u(x, t, \omega),(x, t) \in Q, \omega \in \Omega$, and

$$
\mathcal{A} v \triangleq \sum_{i, j=1}^{n} b_{i j}(x, t, \omega) \frac{\partial^{2} v}{\partial x_{i} \partial x_{j}}(x)+\sum_{i=1}^{n} f_{i}(x, t, \omega) \frac{\partial v}{\partial x_{i}}(x)+\lambda(x, t, \omega) v(x),
$$

where $b_{i j}, f_{i}, x_{i}$ are the components of $b, f$, and $x$. Further,

$$
B_{i} v \triangleq \frac{d v}{d x}(x) \beta_{i}(x, t, \omega)+\bar{\beta}_{i}(x, t, \omega) v(x), \quad i=1, \ldots, N .
$$

We assume that the functions $b(x, t, \omega): \mathbf{R}^{n} \times[0, T] \times \Omega \rightarrow \mathbf{R}^{n \times n}, \beta_{j}(x, t, \omega): \mathbf{R}^{n} \times[0, T] \times \Omega \rightarrow$ $\mathbf{R}^{n}, \bar{\beta}_{i}(x, t, \omega): \mathbf{R}^{n} \times[0, T] \times \Omega \rightarrow \mathbf{R}, f(x, t, \omega): \mathbf{R}^{n} \times[0, T] \times \Omega \rightarrow \mathbf{R}^{n}, \lambda(x, t, \omega): \mathbf{R}^{n} \times[0, T] \times \Omega \rightarrow$ 
$\mathbf{R}$ and $\varphi(x, t, \omega): \mathbf{R}^{n} \times[0, T] \times \Omega \rightarrow \mathbf{R}$ are progressively measurable for any $x \in \mathbf{R}^{n}$ with respect to $\mathcal{F}_{t}$.

We assume that $b(x, t, \omega), f(x, t, \omega), \lambda(x, t, \omega)$ vanish for $(x, t, \omega) \notin D \times[0, T] \times \Omega$.

To proceed further, we assume that Conditions $3.1+3.3$ remain in force throughout this paper.

Condition 3.1 (Coercivity) The matrix $b=b^{\top}$ is symmetric, bounded, and progressively measurable with respect to $\mathcal{F}_{t}$ for all $x$, and there exists a constant $\delta>0$ such that

$$
y^{\top} b(x, t, \omega) y-\frac{1}{2} \sum_{i=1}^{N}\left|y^{\top} \beta_{i}(x, t, \omega)\right|^{2} \geq \delta|y|^{2} \quad \forall y \in \mathbf{R}^{n}, \quad(x, t) \in D \times[0, T], \omega \in \Omega .
$$

Inequality (3.4) means that equation (3.1) is coercive or superparabolic, in the terminology of Rozovskii (1990).

Condition 3.2 Functions $b(x, t, \omega): \mathbf{R}^{n} \times \mathbf{R} \times \Omega \rightarrow \mathbf{R}^{n \times n}, f(x, t, \omega): \mathbf{R}^{n} \times \mathbf{R} \times \Omega \rightarrow \mathbf{R}^{n}$, $\lambda(x, t, \omega): \mathbf{R}^{n} \times \mathbf{R} \times \Omega \rightarrow \mathbf{R}$, are bounded and differentiable in $x$, and

$$
\underset{\omega}{\operatorname{ess} \sup } \operatorname{ess}_{(x, t) \in Q}\left[\left|\frac{\partial b}{\partial x}(x, t, \omega)\right|+\left|\frac{\partial f}{\partial x}(x, t, \omega)\right|+\left|\frac{\partial \lambda}{\partial x}(x, t, \omega)\right|\right]<+\infty .
$$

Condition 3.3 The functions $\beta_{i}(x, t, \omega)$ and $\bar{\beta}_{i}(x, t, \omega)$ are bounded and differentiable in $x$, and $\operatorname{ess}_{\sup } x, t, \omega\left|\frac{\partial \beta_{i}}{\partial x}(x, t, \omega)\right|<+\infty, \operatorname{ess}_{\sup } x, t, \omega\left|\frac{\partial \bar{\beta}_{i}}{\partial x}(x, t, \omega)\right|<+\infty, i=1, \ldots, N$.

We introduce the set of parameters

$$
\begin{aligned}
& \mathcal{P} \triangleq\left(n, D, T \delta, \quad \operatorname{ess} \sup _{x, t, \omega}\left[|b(x, t, \omega)|+|f(x, t, \omega)|+\left|\frac{\partial b}{\partial x}(x, t, \omega)\right|+\left|\frac{\partial f}{\partial x}(x, t, \omega)\right|\right],\right. \\
& \left.\operatorname{ess} \sup _{x, t, \omega, i}\left[\left|\beta_{i}(x, t, \omega)\right|+\left|\bar{\beta}_{i}(x, t, \omega)\right|+\left|\frac{\partial \beta_{i}}{\partial x}(x, t, \omega)\right|+\left|\frac{\partial \bar{\beta}_{i}}{\partial x}(x, t, \omega)\right|\right]\right) .
\end{aligned}
$$

\section{The definition of solution}

Definition 3.4 Let $h_{i} \in X^{0}$ and $\varphi \in X^{-1}$. We say that equations (3.1) are satisfied for $u \in Y^{1}$ if

$$
\begin{aligned}
& u(\cdot, t, \omega)-u(\cdot, r, \omega) \\
& \quad=\int_{r}^{t}(\mathcal{A} u(\cdot, s, \omega)+\varphi(\cdot, s, \omega)) d s+\sum_{i=1}^{N} \int_{r}^{t}\left[B_{i} u(\cdot, s, \omega)+h_{i}(\cdot, s, \omega)\right] d w_{i}(s)
\end{aligned}
$$

for all $r, t$ such that $0 \leq r<t \leq T$, and this equality is satisfied as an equality in $Z_{T}^{-1}$. 
Note that the condition on $\partial D$ is satisfied in the following sense: $u(\cdot, t, \omega) \in H^{1}$ for a.e. $t, \omega$. Further, $u \in Y^{1}$, and the value of $u(\cdot, t, \omega)$ is uniquely defined in $Z_{T}^{0}$ given $t$, by the definitions of the corresponding spaces. The integrals with $d w_{i}$ in (3.5) are defined as elements of $Z_{T}^{0}$. The integral with $d s$ is defined as an element of $Z_{T}^{-1}$. Definition 3.4 requires for (3.1) that this integral must be equal to an element of $Z_{T}^{0}$ in the sense of equality in $Z_{T}^{-1}$.

\section{Existence theorems and fundamental inequalities for forward equations}

The following Lemma combines the first and the second fundamental inequalities and related existence result for forward SPDEs (the cases when $k=-1$ and $k=1$ respectively). It is an analog of the so-called "energy inequalities", or "the fundamental inequalities" known for deterministic parabolic equations (Ladyzhenskaya et al (1969)).

Lemma 3.5 Let either $k=-1$ or $k=0$. Assume that Conditions [3.1, 3.2, and 3.3, are satisfied. In addition, assume that if $k=0$, then $\beta_{i}(x, t, \omega)=0$ for $x \in \partial D, i=1, \ldots, N$. Let $\varphi \in X^{k}(s, T)$ and $\Phi \in Z_{s}^{k+1}$. Then problem (3.1) has an unique solution $u$ in the class $Y^{1}(s, T)$, and the following analog of the first fundamental inequality is satisfied:

$$
\|u\|_{Y^{k+2}(s, T)} \leq c\left(\|\varphi\|_{X^{k}(s, T)}+\|\Phi\|_{Z_{s}^{k+1}}+\sum_{i=1}^{N}\left\|h_{i}\right\|_{X^{k+1}(s, T)}\right),
$$

where $c=c(\mathcal{P})$ is a constant that depends on $\mathcal{P}$ only.

The result of Lemma 3.5 for $k=-1$ is well known for long time (see, e.g., Rozovskii (1990), Ch. 3.4.1). The result for $k=0$ was obtained in Dokuchaev (2005).

Note that $Y^{k+2}(s, T)=X^{k+2}(s, T) \cap \mathcal{C}^{k+1}(s, T)$, hence the solution $u=u(\cdot, t)$ is continuous in $t$ in $L_{2}\left(\Omega, \mathcal{F}, \mathbf{P}, H^{k+1}\right)$.

Introduce operators $L(s, T): X^{-1}(s, T) \rightarrow Y^{1}(s, T), \mathcal{M}_{i}(s, T): X^{0}(s, T) \rightarrow Y^{1}(s, T)$, and $\mathcal{L}(s, T): Z_{s}^{0} \rightarrow Y^{1}(s, T)$, such that

$$
u=L(s, T) \varphi+\mathcal{L}(s, T) \Phi+\sum_{i=1}^{N} \mathcal{M}_{i}(s, T) h_{i},
$$

where $u$ is the solution in $Y^{1}(s, T)$ of problem (3.1). These operators are linear and continuous; it follows immediately from Lemma 3.5. We shall denote by $L, \mathcal{M}_{i}$, and $\mathcal{L}$, the operators $L(0, T)$, $\mathcal{M}_{i}(0, T)$, and $\mathcal{L}(0, T)$, correspondingly. 


\section{Backward equations}

Introduce the operators being formally adjoint to the operators $\mathcal{A}$ and $B_{i}$ :

$$
\begin{aligned}
& \mathcal{A}^{*} v=\sum_{i, j=1}^{n} \frac{\partial^{2}}{\partial x_{i} \partial x_{j}}\left(b_{i j}(x, t, \omega) v(x)\right)-\sum_{i=1}^{n} \frac{\partial}{\partial x_{i}}\left(f_{i}(x, t, \omega) v(x)\right)+\lambda(x, t, \omega) v(x), \\
& B_{i}^{*} v=-\sum_{i=1}^{n} \frac{\partial}{\partial x_{i}}\left(\beta_{i}(x, t, \omega) v(x)\right)+\bar{\beta}_{i}(x, t, \omega) v(x) .
\end{aligned}
$$

Consider the boundary value problem in $Q$

$$
\begin{gathered}
d_{t} p+\left(\mathcal{A}^{*} p+\sum_{i=1}^{N} B_{i}^{*} \chi_{i}+\xi\right) d t=\sum_{i=1}^{N} \chi_{i} d w_{i}(t) \\
\left.p\right|_{t=T}=\Psi,\left.\quad p(x, t, \omega)\right|_{x \in \partial D}=0 .
\end{gathered}
$$

Definition 4.1 We say that equation (4.1) is satisfied for $p \in Y^{1}, \Psi \in Z_{T}^{0}, \chi_{i} \in X^{0}$ if

$$
p(\cdot, t)=\Psi+\int_{t}^{T}\left(\mathcal{A}^{*} p(\cdot, s)+\sum_{i=1}^{N} B_{i}^{*} \chi_{i}(\cdot, s)+\xi(\cdot, s)\right) d s-\sum_{i=1}^{N} \int_{t}^{T} \chi_{i}(\cdot, s) d w_{i}(s)
$$

for any $t \in[0, T]$. The equality here is assumed to be an equality in the space $Z_{T}^{-1}$.

Theorem 4.2 For any $\xi \in X^{-1}$ and $\Psi \in Z_{T}^{0}$, there exists a pair $(p, \chi)$, such that $p \in Y^{1}$, $\chi=\left(\chi_{1}, \ldots, \chi_{N}\right), \chi_{i} \in X^{0}$ and (4.1) is satisfied. This pair is uniquely defined, and the following analog of the first fundamental inequality is satisfied:

$$
\|p\|_{Y^{1}}+\sum_{i=1}^{N}\left\|\chi_{i}\right\|_{X^{0}} \leq c\left(\|\xi\|_{X^{-1}}+\|\Psi\|_{Z_{T}^{0}}\right)
$$

where $c=c(\mathcal{P})>0$ is a constant that does not depend on $\xi$ and $\Psi$. In addition,

$$
p=L^{*} \xi+\left(\delta_{T} L\right)^{*} \Psi, \quad \chi_{i}=\mathcal{M}_{i}^{*} \xi+\left(\delta_{T} \mathcal{M}_{i}\right)^{*} \Psi, \quad p(\cdot, 0)=\mathcal{L}^{*} \xi+\left(\delta_{T} \mathcal{L}\right)^{*} \Psi
$$

where $L^{*}: X^{-1} \rightarrow X^{1}, \mathcal{M}_{i}^{*}: X^{0} \rightarrow X^{0},\left(\delta_{T} L\right)^{*}: Z_{0}^{0} \rightarrow X^{1},\left(\delta_{T} \mathcal{M}_{i}\right)^{*}: Z_{0}^{0} \rightarrow X^{0}$, and $\left(\delta_{T} \mathcal{L}\right)^{*}:$ $Z_{T}^{0} \rightarrow Z_{0}^{0}$, are the operators that are adjoint to the operators $L: X^{-1} \rightarrow X^{1}, \mathcal{M}_{i}: X^{0} \rightarrow X^{1}$, $\delta_{T} \mathcal{M}_{i}: X^{-1} \rightarrow Z_{T}^{0}, \delta_{T} \mathcal{M}_{i}: X^{0} \rightarrow Z_{T}^{0}$, and $\delta_{T} \mathcal{L}: Z_{0}^{0} \rightarrow Z_{T}^{0}$, respectively.

An example of application of duality established in this theorem is given in Theorem 6.1. 


\section{$5 \quad$ Proof of Theorem 4.2}

Theorem 4.2 extends Theorem 4.1 from Dokuchaev (1992) and Theorem 3.2 in Dokuchaev (2003) for the case of non-zero $\Psi, \beta_{i}, \bar{\beta}_{i}$. Apparently, the extension required was non-trivial and, as can be seen from the proof below, required solid efforts. We shall prove Theorem 4.2 using the following steps: first, we obtain some decomposition results for the basic operators, the the proof for the case when the coefficients of $\mathcal{A}$ are $\mathcal{F}_{0}$-measurable and $B_{i} \equiv 0$, then we consider the case when $\mathcal{A}$ is of the general form and $B_{i} \equiv 0$, and then we consider the general case.

\subsection{Decomposition of operators $L$ and $\mathcal{M}_{i}$}

Our method of proof is based on decomposition of the operators to superpositions of simpler operators.

Definition 5.1 Define operators $K: Z_{0}^{0} \rightarrow Y^{1}, \mathcal{Q}_{0}: X^{-1} \rightarrow Y^{1}, \mathcal{Q}_{i}: X^{0} \rightarrow Y^{1}, i=1, \ldots, N$, as the operators $\mathcal{L}: Z_{0}^{0} \rightarrow Y^{1}, L: X^{-1} \rightarrow Y^{1}, \mathcal{M}_{i}: X^{0} \rightarrow Y^{1}, i=1, \ldots, N$, considered for the case when $B_{i}=0$ for all $i$.

By Lemma 3.5, these linear operators are continuous. It follows from the definitions that

$$
K \Phi+\mathcal{Q}_{0} \eta+\sum_{i=1}^{N} \mathcal{Q}_{i} h_{i}=V
$$

where $\eta \in X^{-1}, \Phi \in Z_{0}^{0}$, and $h_{i} \in X^{0}$, and where $V$ is the solution of the problem

$$
\begin{gathered}
d_{t} V=(\mathcal{A} V+\eta) d t+\sum_{i=1}^{N} h_{i} d w_{i}(t), \\
\left.V\right|_{t=0}=\Phi,\left.\quad V(x, t, \omega)\right|_{x \in \partial D}=0 .
\end{gathered}
$$

Define the operators

$$
P \triangleq \sum_{i=1}^{N} \mathcal{Q}_{i} B_{i}, \quad P^{*} \triangleq \sum_{i=1}^{N} B_{i}^{*} \mathcal{Q}_{i}^{*} .
$$

Clearly, the operator $P: X^{1} \rightarrow X^{1}$ is continuous, and $P^{*}: X^{-1} \rightarrow X^{-1}$ is its adjoint operator. Hence the operator $P^{*}: X^{-1} \rightarrow X^{-1}$ is continuous. Since the operators $\mathcal{Q}_{i}^{*}:\left(\mathcal{C}^{0}\right)^{*} \rightarrow X^{0}$ and $B_{i}^{*}: X^{0} \rightarrow X^{-1}$ are continuous, it follows that the operator $P^{*}:\left(\mathcal{C}^{0}\right)^{*} \rightarrow X^{-1}$ is continuous.

Let

$$
P_{0} \triangleq \delta_{T} \sum_{i=1}^{N} \mathcal{Q}_{i} B_{i}, \quad P_{0}^{*} \triangleq \sum_{i=1}^{N} B_{i}^{*}\left(\delta_{T} \mathcal{Q}_{i}\right)^{*}
$$


Lemma 5.2 The operator $(I-P)^{-1}: X^{1} \rightarrow X^{1}$ is continuous, and

$$
\begin{aligned}
& L=(I-P)^{-1} \mathcal{Q}_{0}, \quad \mathcal{L}=(I-P)^{-1} K, \quad \mathcal{M}_{i}=(I-P)^{-1} \mathcal{Q}_{i} \\
& \delta_{T} L=P_{0}(I-P)^{-1} \mathcal{Q}_{0}+\delta_{T} \mathcal{Q}_{0}, \quad \delta_{T} \mathcal{M}_{i}=P_{0}(I-P)^{-1} \mathcal{Q}_{i}+\delta_{T} \mathcal{Q}_{i}
\end{aligned}
$$

$i=1, \ldots, N$. The operator $\left(I-P^{*}\right)^{-1}: X^{-1} \rightarrow X^{-1}$ is also continuous, and

$$
\begin{aligned}
& L^{*}=\mathcal{Q}_{0}^{*}\left(I-P^{*}\right)^{-1}, \quad \mathcal{L}^{*}=K^{*}\left(I-P^{*}\right)^{-1}, \quad \mathcal{M}_{i}^{*}=\mathcal{Q}_{i}^{*}\left(I-P^{*}\right)^{-1}, \\
& \left(\delta_{T} L\right)^{*}=\mathcal{Q}_{0}^{*}\left(I-P^{*}\right)^{-1} P_{0}^{*}+\left(\delta_{T} \mathcal{Q}_{0}\right)^{*}, \quad\left(\delta_{T} \mathcal{M}_{i}\right)^{*}=\mathcal{Q}_{i}^{*}\left(I-P^{*}\right)^{-1} P_{0}^{*}+\left(\delta_{T} \mathcal{Q}_{i}\right)^{*}
\end{aligned}
$$

Proof. If $\gamma \in X^{1}$, then the solution of problem (3.1) with $\Phi=0, \varphi=0$, and $h_{i} \triangleq B_{i} \gamma$, can be represented as

$$
V=\sum_{i=1}^{n} \mathcal{Q}_{i} B_{i}(\gamma+V)=P(\gamma+V)
$$

By Lemma 3.5, $\|V\|_{Y^{1}} \leq$ const $\|\gamma\|_{X^{1}}$. Hence the problem

$$
V=P(\gamma+V)
$$

has the unique solution $V \in Y^{1}$. Define the operator $\widehat{P}: X^{1} \rightarrow Y^{1}$ such that $V=\widehat{P} \gamma=P(\gamma+V)$ is the solution of problem (5.5).

Let $\gamma \in X^{1}$ be given. Set $V \triangleq \widehat{P} \gamma, h \triangleq \gamma+V$. Clearly, $\|h\|_{X^{1}} \leq \operatorname{const}\left(\|\gamma\|_{X^{1}}+\|\zeta\|_{X^{1}}\right)$ ?. By the definitions,

$$
V=P h, \quad \gamma=h-P h, \quad h=(I-P)^{-1} \gamma
$$

We have noticed already that $\|V\|_{Y^{1}} \leq$ const $\|\gamma\|_{X^{1}}$. Hence $\|h\|_{X^{1}} \leq$ const $\|\gamma\|_{X^{1}}$, and the operator $(I-P)^{-1}: X^{1} \rightarrow X^{1}$ is continuous. Therefore, the adjoint operator $\left(I-P^{*}\right)^{-1}$ : $X^{-1} \rightarrow X^{-1}$ is also continuous.

By the definitions, the solution of problem (3.6) has the form

$$
V=L \varphi+\mathcal{L} \Phi+\sum_{i=1}^{N} \mathcal{M}_{i} h_{i}=\mathcal{Q}_{0} \varphi+\sum_{i=1}^{n} \mathcal{Q}_{i}\left[B_{i} V+h_{i}\right]+K \Phi=\mathcal{Q}_{0} \varphi+\sum_{i=1}^{N} \mathcal{Q}_{i} h_{i}+P V+K \Phi
$$

for any $\varphi \in X^{-1}, h_{i} \in X^{0}$, and $\Phi \in Z_{0}^{0}$. Then the first three equations in (5.3) follow. Clearly,

$$
(I-P)^{-1}=P(I-P)^{-1}-I
$$

Hence the last two equations in (5.3) follow. Therefore, all equations (5.4) for the adjoint operators follow. This completes the proof of Lemma 5.2 . 
We found that the operator $\left(I-P^{*}\right)^{-1}: X^{-1} \rightarrow X^{-1}$ is continuous. In addition, Theorem 3.2 from p. 467 of Dokuchaev (1992) stated that the operator $P^{*}: X^{0} \rightarrow X^{0}$ is continuous under some additional technical conditions. The question arises if the operator $(I-P)^{-1}: X^{0} \rightarrow X^{0}$ is continuous. If this is true, then, by Lemma 5.2, the operators $L^{*}: X^{0} \rightarrow Y^{2}, \mathcal{M}_{i}^{*}: X^{0} \rightarrow X^{1}$ are continuous; in that case, an analog of the second fundamental inequality holds for backward equations, and existence has place for solutions $p \in X^{2}$ and $\chi_{i} \in X^{1}$.

\subsection{Proof of Theorem 4.2 for $B_{i} \equiv 0$ and $\mathcal{F}_{0}$-measurable $(b, f, \lambda)$}

The following lemma extends Theorem 4.1 from Dokuchaev (1992) for the case of non-zero $\Psi$.

Lemma 5.3 Theorem 4.2 holds under additional assumptions that the function $\mu=(b, f, \lambda)$ is such that $\mu(x, t, \omega)$ is $\mathcal{F}_{0}$-measurable for all $(x, t)$, and $\beta_{i} \equiv 0, \bar{\beta}_{i} \equiv 0$ for $i=1, \ldots, N$.

Proof. It suffices to prove that, for any $\xi \in X^{-1}$, there exists a pair $(p, \chi)$, with $p \in Y^{1}$, and $\chi=\left(\chi_{1}, \ldots, \chi_{N}\right), \chi_{i} \in X^{0}$, such that

$$
\begin{array}{r}
d_{t} p+\left(\mathcal{A}^{*} p+\xi\right) d t=\sum_{i=1}^{N} \chi_{i} d w_{i}(t), \\
\left.p\right|_{t=T}=\Psi,\left.\quad p(x, t, \omega)\right|_{x \in \partial D}=0,
\end{array}
$$

and that

$$
p=\mathcal{Q}_{0}^{*} \xi+\left(\delta_{T} \mathcal{Q}_{0}\right)^{*} \Psi, \quad \chi_{i}=\mathcal{Q}_{i}^{*} \xi+\left(\delta_{T} \mathcal{Q}_{i}\right)^{*} \Psi, \quad p(., 0)=K^{*} \xi+\left(\delta_{T} K\right)^{*} \Psi,
$$

where $\mathcal{Q}_{0}^{*}: X^{-1} \rightarrow X^{1}, \mathcal{Q}_{i}^{*}: X^{-1} \rightarrow X^{0},\left(\delta_{T} \mathcal{Q}_{0}\right)^{*}: Z_{T}^{0} \rightarrow X^{1},\left(\delta_{T} \mathcal{Q}_{i}\right) *: Z_{T}^{0} \rightarrow X^{0}, i=1, \ldots, N$, and $K^{*}: X^{-1} \rightarrow Z_{0}^{0}$, are the operators that are adjoint for the operators $\mathcal{Q}_{0}: X^{-1} \rightarrow X^{1}$, $\mathcal{Q}_{i}: X^{0} \rightarrow X^{1}, \delta_{T} \mathcal{Q}_{i}: X^{0} \rightarrow Z_{T}^{0}$, and $K^{*}: Z_{0}^{0} \rightarrow X^{1}$, correspondingly. We know that these operators are continuous.

We interpret the solution of problem (5.6) similarly to Definition 4.1.

Let $\bar{p}$ be the solution of the problem

$$
\begin{aligned}
& \frac{\partial \bar{p}}{\partial t}+\mathcal{A}^{*} \bar{p}=-\xi \\
& \left.\bar{p}\right|_{t=T}=\Psi,\left.\quad \bar{p}(x, t, \omega)\right|_{x \in \partial D}=0 .
\end{aligned}
$$

Clearly, $\bar{p} \in \bar{X}^{1} \cap C\left([0, T] ; Z_{T}^{0}\right)$.

Let $p \triangleq \mathcal{E} \bar{p}$, and let $\varphi \in L_{\infty}\left(\Omega, \mathbf{P}, \mathcal{F}_{T}, C(Q)\right), \Phi \in L_{2}\left(\Omega, \mathbf{P}, \mathcal{F}_{0}, C^{1}(D)\right) \cap Z_{0}^{1}$. Then $\bar{p}$ and its derivatives presented in (5.8) belong to $L_{\infty}\left(\Omega, \mathbf{P}, \mathcal{F}_{T}, C(Q)\right)$. We have that if $V=\mathcal{Q}_{0} \varphi+K \Phi$ 
then

$$
\begin{aligned}
& \frac{\partial V}{\partial t}=\mathcal{A} V+\varphi \\
& \left.V\right|_{t=0}=\Phi,\left.\quad V(x, t, \omega)\right|_{x \in \partial D}=0 .
\end{aligned}
$$

Hence

$$
\begin{gathered}
(\bar{p}(\cdot, T), V(\cdot, T))_{Z_{T}^{0}}-(\bar{p}(\cdot, 0), V(\cdot, 0))_{Z_{T}^{0}}=\left(-\mathcal{A}^{*} \bar{p}-\xi, V\right)_{\bar{X}^{0}}+(\bar{p}, \mathcal{A} V+\varphi)_{\bar{X}^{0}} . \\
(\Psi, V(\cdot, T))_{Z_{T}^{0}}-(\bar{p}(\cdot, 0), \Phi)_{Z_{T}^{0}}=(-\xi, V)_{\bar{X}^{0}}+(\bar{p}, \varphi)_{\bar{X}^{0}} .
\end{gathered}
$$

Hence

$$
(\Psi, V(\cdot, T))_{Z_{T}^{0}}-(p(\cdot, 0), \Phi)_{Z_{0}^{0}}=(-\xi, V)_{X^{0}}+(p, \varphi)_{X^{0}}
$$

i.e.,

$$
\begin{aligned}
(p, \varphi)_{X^{0}}+(p(\cdot, 0), \Phi)_{Z_{0}^{0}} & =(\Psi, V(\cdot, T))_{Z_{T}^{0}}+(\xi, V)_{X^{0}} \\
& =\left(\Psi, \delta_{T} \mathcal{Q}_{0} \varphi+\delta_{T} K \Phi\right)_{Z_{T}^{0}}+\left(\xi, \mathcal{Q}_{0} \varphi+K \Phi\right)_{X^{0}}
\end{aligned}
$$

Then $p=\mathcal{Q}_{0}^{*} \xi+\left(\delta_{T} \mathcal{Q}_{0}\right)^{*} \Psi$ and $p(., 0)=K^{*} \xi+\left(\delta_{T} K\right)^{*} \Psi$.

Remark 5.4 Up to this point, we didn't use the assumption that the coefficients $\mathcal{A}$ are $\mathcal{F}_{0}$ measurable; all previous reasons are valid for the general $\mathcal{A}$ as well.

Further, by Clark's Theorem, there exist functions $\gamma_{i}(\cdot, t, \cdot) \in X^{0}, \gamma_{\Psi i} \in X^{0}$, and $\gamma_{\xi i}(\cdot, t, \cdot) \in$ $X^{0}$, such that

$$
\begin{gathered}
\bar{p}(x, t, \omega)=\mathbf{E} \bar{p}(x, t, \omega)+\sum_{i=1}^{N} \int_{0}^{T} \gamma_{i}(x, t, s, \omega) d w_{i}(s), \\
\bar{\Psi}(x, t, \omega)=\mathbf{E} \Psi(x, \omega)+\sum_{i=1}^{N} \int_{0}^{T} \gamma_{\Psi i}(x, s, \omega) d w_{i}(s), \\
\xi(x, t, \omega)=\mathbf{E} \xi(x, t, \omega)+\sum_{i=1}^{N} \int_{0}^{T} \gamma_{\xi i}(x, t, s, \omega) d w_{i}(s) .
\end{gathered}
$$

Moreover, it follows that $\mathcal{D} g_{i}(\cdot, t, \cdot) \in X^{0}$, where either $\mathcal{D} \gamma=\partial \gamma / \partial t$ or $\mathcal{D} \gamma=\mathcal{A}^{*} \gamma=\Delta \gamma$, and

$$
\mathcal{D} \bar{p}(x, t, \omega)=\mathbf{E} \mathcal{D} \bar{p}(x, t, \omega)+\sum_{i=1}^{N} \int_{0}^{T} \mathcal{D} \gamma_{i}(x, t, s, \omega) d w_{i}(s) .
$$

By (5.8),

$$
\begin{aligned}
& \frac{\partial \gamma_{i}}{\partial t}(\cdot, t, s, \omega)+\mathcal{A}^{*} \gamma_{i}(\cdot, t, s, \omega)=-\gamma_{\xi i}(\cdot, t, s, \omega), \\
& \gamma_{i}(x, T, s, \omega)=\gamma_{\Psi i}(x, s, \omega),\left.\quad \gamma_{i}(x, t, s, \omega)\right|_{x \in \partial D}=0 .
\end{aligned}
$$


It follows that

$$
\sup _{t \in[s, T]} \int_{D}\left|\gamma_{i}(x, t, s, \omega)\right|^{2} d x \leq c\left[\int_{s}^{T}\left\|\gamma_{\xi i}(\cdot, t, s, \omega)\right\|_{H^{-1}}^{2} d t+\int_{D}\left|\gamma_{\Psi i}(x, s, \omega)\right|^{2} d x\right],
$$

where $c=c(T, n, D)>0$ is a constant. Hence

$$
\int_{D}\left|\gamma_{i}(x, s, s, \omega)\right|^{2} d x \leq c\left[\int_{s}^{T}\left\|\gamma_{\xi i}(\cdot, t, s, \omega)\right\|_{H^{-1}}^{2} d t+\int_{D}\left|\gamma_{\Psi i}(x, s, \omega)\right|^{2} d x\right] .
$$

Let

$$
\chi_{i}(x, t, \omega) \triangleq \gamma_{i}(x, t, t, \omega)
$$

It follows that

$$
\left\|\chi_{i}\right\|_{X^{0}} \leq c\left(\|\xi\|_{X^{-1}}+\|\Psi\|_{Z_{T}^{0}}\right) .
$$

Let us show that the pair $(p, \chi), \chi=\left(\chi_{1}, \ldots, \chi_{N}\right)$, is such that (5.6) is satisfied. Clearly,

$$
\bar{p}(x, t, \omega)=p(x, t, \omega)+\sum_{i=1}^{N} \int_{t}^{T} \gamma_{i}(x, t, s, \omega) d w_{i}(s),
$$

and

$$
\bar{p}(\cdot, t)=\Psi+\int_{t}^{T}\left(\mathcal{A}^{*} \bar{p}(\cdot, s)+\xi(\cdot, s)\right) d s
$$

Hence

$$
\begin{aligned}
& p(\cdot, t)=\Psi+\int_{t}^{T}\left(\mathcal{A}^{*} p(\cdot, s)+\xi(\cdot, s)\right) d s \\
& +\sum_{i=1}^{N}\left[-\int_{t}^{T} \gamma_{i}(\cdot, t, s) d w_{i}(s)+\int_{t}^{T} d s \int_{s}^{T}\left[\mathcal{A}^{*} \gamma_{i}(\cdot, s, r)+\gamma_{\xi i}(\cdot, s, r)\right] d w_{i}(r)\right] \\
& =\Psi+\int_{t}^{T}\left(\mathcal{A}^{*} p(\cdot, s)+\xi(\cdot, s)\right) d s \\
& +\sum_{i=1}^{N}\left[-\int_{t}^{T} \gamma_{i}(\cdot, t, s) d w_{i}(s)+\int_{t}^{T} d w_{i}(r) \int_{t}^{r}\left[\mathcal{A}^{*} \gamma_{i}(\cdot, s, r)+\gamma_{\xi i}(\cdot, s, r)\right] d s\right] \\
& =\Psi+\int_{t}^{T}\left(\mathcal{A}^{*} p(\cdot, s)+\xi(\cdot, s)\right) d s \\
& +\sum_{i=1}^{N} \int_{t}^{T} d w_{i}(s)\left[-\gamma_{i}(\cdot, t, s)+\int_{t}^{s}\left[\mathcal{A}^{*} \gamma_{i}(\cdot, r, s)+\gamma_{\xi i}(\cdot, r, s)\right] d r\right] \\
& =\Psi+\int_{t}^{T}\left(\mathcal{A}^{*} p(\cdot, s)+\xi(\cdot, s)\right) d s-\int_{t}^{T} \sum_{i=1}^{N} \chi_{i}(\cdot, s) d w_{i}(s),
\end{aligned}
$$


since

$$
\gamma_{i}(\cdot, t, s)-\int_{t}^{s}\left[\mathcal{A}^{*} \gamma_{i}(\cdot, r, s)+\gamma_{\xi i}(\cdot, r, s)\right] d r=\gamma_{i}(\cdot, s, s)=\chi_{i}(\cdot, s) .
$$

We have that if $V=\mathcal{Q}_{i} h$, where $h \in X^{0}$, then

$$
\begin{aligned}
& d_{t} V=\mathcal{A} V d t+h d w_{i}(t), \\
& \left.V\right|_{t=0}=0,\left.\quad V(x, t, \omega)\right|_{x \in \partial D}=0 .
\end{aligned}
$$

Hence

$$
(p(\cdot, T), V(\cdot, T))_{Z_{T}^{0}}-(p(\cdot, 0), V(\cdot, 0))_{Z_{T}^{0}}=\left(-\mathcal{A}^{*} \bar{p}-\xi, V\right)_{X^{0}}+(p, \mathcal{A} V)_{X^{0}}+\left(\chi_{i}, h\right)_{X^{0}}
$$

i.e., $(\Psi, V(\cdot, T))_{Z_{T}^{0}}=(-\xi, V)_{X^{0}}+\left(\chi_{i}, h\right)_{X^{0}}$. It follows that

$$
\left(\chi_{i}, h\right)_{X^{0}}=\left(\Psi, \delta_{T} \mathcal{Q}_{i} h\right)_{Z_{T}^{0}}+\left(\xi, \mathcal{Q}_{i} h\right)_{X^{0}} \quad \forall h \in X^{0}
$$

Hence function (5.12) is

$$
\chi_{i}=\mathcal{Q}_{i}^{*} \xi+\left(\delta_{T} \mathcal{Q}_{i}\right)^{*} \Psi .
$$

This completes the proof of Lemma 5.3 .

\subsection{Proof of Theorem 3.6 for $B_{i} \equiv 0$}

Lemma 5.5 Theorem [3.6 holds under additional assumptions that $\beta_{i} \equiv 0, \bar{\beta}_{i} \equiv 0$ for $i=$ $1, \ldots, N$, i.e., when $B_{i}=0$ for all $i$.

Proof. It suffices to prove that (5.7) holds. Introduce the operators $\tilde{\mathcal{A}}$ and $\tilde{\mathcal{A}}^{*}$ such that

$$
\tilde{\mathcal{A}} v=\tilde{\mathcal{A}}^{*} \triangleq \Delta v=\sum_{i=1}^{n} \frac{\partial^{2} v}{\partial x_{i}^{2}},
$$

where $\Delta$ is the Laplace operator,Denote by $\tilde{\mathcal{Q}}_{i}, \tilde{K}, \tilde{\mathcal{Q}}_{i}^{*}$ and $\tilde{K}^{*}$, the operators, defined similarly $\mathcal{Q}_{i}, K, \mathcal{Q}_{i}^{*}, K^{*}$, but with substituting $\mathcal{A}=\tilde{\mathcal{A}}$. Introduce the operators

$$
\mathcal{U} \triangleq \tilde{\mathcal{Q}}_{0}(\tilde{\mathcal{A}}-\mathcal{A}), \quad \mathcal{U}_{\Delta} \triangleq \tilde{\mathcal{Q}}_{0}^{*}\left(\tilde{\mathcal{A}}^{*}-\mathcal{A}^{*}\right), \quad
$$

Clearly, the operators $\mathcal{A}: X^{1} \rightarrow X^{-1}$ and $\mathcal{A}^{*}: X^{1} \rightarrow X^{-1}$ are continuous. By Lemma 3.5, the operator $\mathcal{Q}_{0}: X^{-1} \rightarrow X^{1}$ is continuous. Hence the adjoint operator $\mathcal{Q}_{0}^{*}: X^{-1} \rightarrow X^{1}$ is continuous, the operators $\mathcal{U}: X^{1} \rightarrow X^{1}, \mathcal{U}_{\Delta}: X^{1} \rightarrow X^{1}$ are both continuous, and the adjoint operators $\mathcal{U}^{*}: X^{-1} \rightarrow X^{-1}$ and $\mathcal{U}_{\Delta}^{*}: X^{-1} \rightarrow X^{-1}$ are continuous, where $\mathcal{U}_{\Delta}^{*}=(\tilde{A}-\mathcal{A}) \tilde{\mathcal{Q}}_{0}$ and $\mathcal{U}^{*} \triangleq\left(\tilde{A}^{*}-\mathcal{A}^{*}\right) \tilde{\mathcal{Q}}_{0}^{*}$. 
1. Let us prove that the operator $\left(I+\mathcal{U}_{\Delta}\right)^{-1} \tilde{\mathcal{Q}}_{0}^{*}: X^{1} \rightarrow X^{1}$ is continuous. First, we shall prove that the operator $(I+\mathcal{U})^{-1} \tilde{\mathcal{Q}}_{0}: X^{-1} \rightarrow X^{-1}$ is continuous.

Let $\eta \in X^{-1}, h_{i} \in X^{0}$, and $\Phi \in Z_{0}^{0}$. By Lemma 3.5, the boundary value problem

$$
\begin{aligned}
& d_{t} z=(\tilde{A} z+(A-\tilde{A}) z+\eta) d t+\sum_{i=1}^{N} h_{i} d w_{i}(t)=(A z+\eta) d t+\sum_{i=1}^{N} h_{i} d w_{i}(t), \\
& \left.z\right|_{t=0}=\Phi,\left.\quad z(x, t, \omega)\right|_{x \in \partial D}=0
\end{aligned}
$$

has the unique solution $z \in Y^{1}$, and $\|z\|_{Y^{1}} \leq \operatorname{const}\left(\|\eta\|_{X^{-1}}+\sum_{i}\left\|h_{i}\right\|_{X^{0}}+\|\Phi\|_{Z_{0}^{0}}\right)$. By the definitions of the corresponding operators, the solution $z$ of the equation (5.15) is

$$
z=\mathcal{Q}_{0} \eta+\sum_{i} \mathcal{Q}_{i} h_{i}+K \Phi=\tilde{\mathcal{Q}}_{0}(\eta+(\mathcal{A}-\tilde{\mathcal{A}}) z)+\sum_{i} \tilde{\mathcal{Q}}_{i} h_{i}+\tilde{K} \Phi
$$

or

$$
z=\mathcal{Q}_{0} \eta+\sum_{i=1}^{N} \mathcal{Q}_{i} h_{i}+K \Phi=(I+\mathcal{U})^{-1}\left(\tilde{\mathcal{Q}}_{0} \eta+\sum_{i=1}^{N} \tilde{\mathcal{Q}}_{i} h_{i}+\tilde{K} \Phi\right)
$$

Hence the operator $(I+\mathcal{U})^{-1} \tilde{\mathcal{Q}}_{0}: X^{-1} \rightarrow X^{1}$ is continuous.

Further, note that the range of the operator $\tilde{\mathcal{Q}}_{0}\left(X^{-1}\right)$ contains all smooth functions from $X^{1}$, therefore, the range is dense in $X^{-1}$. Clearly, the equality $\tilde{\mathcal{Q}}_{0} y=0$ implies that $y=0$. Let $y \in \tilde{\mathcal{Q}}_{0}\left(X^{-1}\right)$ and $y=\tilde{\mathcal{Q}}_{0} z$. Then the equality $\tilde{\mathcal{Q}}_{0}\left(I+\mathcal{U}_{\Delta}^{*}\right)^{-1} x=y$ implies equalities

$$
\begin{array}{r}
\left(I+\mathcal{U}_{\Delta}^{*}\right)^{-1} x=z, \quad\left(I+\mathcal{U}_{\Delta}^{*}\right) z=x, \quad \tilde{\mathcal{Q}}_{0}^{-1} y+\mathcal{U}_{\Delta}^{*} \tilde{\mathcal{Q}}_{0}^{-1} y=x, \quad \tilde{\mathcal{Q}}_{0}^{-1} y+(\tilde{A}-A) y=x \\
\tilde{\mathcal{Q}}_{0}^{-1} y+\tilde{\mathcal{Q}}_{0}^{-1} \mathcal{U} y=x, \quad \tilde{\mathcal{Q}}_{0}^{-1}(I+\mathcal{U}) y=x
\end{array}
$$

i.e.

$$
\tilde{\mathcal{Q}}_{0}\left(I+\mathcal{U}_{\Delta}^{*}\right)^{-1}=(I+\mathcal{U})^{-1} \tilde{\mathcal{Q}}_{0}
$$

This and continuity of the operator $(I+\mathcal{U})^{-1} \tilde{\mathcal{Q}}_{0}: X^{-1} \rightarrow X^{1}$ imply that the operator $\tilde{\mathcal{Q}}_{0}(I+$ $\left.\mathcal{U}_{\Delta}^{*}\right)^{-1}: X^{-1} \rightarrow X^{1}$ is continuous. Therefore, the adjoint operator $\left(I+\mathcal{U}_{\Delta}\right)^{-1} \tilde{\mathcal{Q}}_{0}^{*}: X^{-1} \rightarrow X^{1}$ is continuous.

2. Let us establish the connection between (5.6) and the operators $\mathcal{Q}_{i}, i=0, \ldots, N$. It was shown above that the operator $\left(I+\mathcal{U}_{\Delta}\right)^{-1} \tilde{\mathcal{Q}}_{0}^{*}: X^{-1} \rightarrow X^{1}$ is continuous. By (5.15), it follows that

$$
\mathcal{Q}_{0} \xi=(I+\mathcal{U})^{-1} \tilde{\mathcal{Q}}_{0} \xi, \quad \mathcal{Q}_{i} h_{i}=(I+\mathcal{U})^{-1} \tilde{\mathcal{Q}}_{i} h_{i}, \quad i=1, \ldots, N, \quad K \Phi=(I+\mathcal{U})^{-1} \tilde{K} \Phi
$$


for all $\eta \in X^{-1}, h_{i} \in X^{0}$, and $\Phi \in Z_{0}^{0}$. Hence

$$
\mathcal{Q}_{0}=(I+\mathcal{U})^{-1} \tilde{\mathcal{Q}}_{0}, \quad \mathcal{Q}_{i}=(I+\mathcal{U})^{-1} \tilde{\mathcal{Q}}_{i}, \quad i=1, \ldots, N, \quad K=(I+\mathcal{U})^{-1} \tilde{K}
$$

and

$$
\mathcal{Q}_{0}^{*}=\tilde{\mathcal{Q}}_{0}^{*}\left(I+\mathcal{U}^{*}\right)^{-1}, \quad \mathcal{Q}_{i}^{*}=\tilde{\mathcal{Q}}_{i}^{*}\left(I+\mathcal{U}^{*}\right)^{-1}, \quad i=1, \ldots, N, \quad K=\tilde{K}^{*}\left(I+\mathcal{U}^{*}\right)^{-1}
$$

By (15.16), it follows that

$$
\left(I+\mathcal{U}_{\Delta}\right)^{-1} \tilde{\mathcal{Q}}_{0}^{*}=\tilde{\mathcal{Q}}_{0}^{*}\left(I+\mathcal{U}^{*}\right)^{-1}
$$

Set

$$
p \triangleq\left(I+\mathcal{U}_{\Delta}\right)^{-1}\left[\tilde{\mathcal{Q}}_{0}^{*} \xi+\left(\delta_{T} \tilde{\mathcal{Q}}_{0}\right)^{*} \Psi\right] .
$$

Clearly, $\left(I+\mathcal{U}_{\Delta}\right) p=\tilde{\mathcal{Q}}_{0}^{*} \xi+\left(\delta_{T} \tilde{\mathcal{Q}}_{0}\right)^{*} \Psi$, i.e.

$$
p=\tilde{\mathcal{Q}}_{0}^{*} \xi-\mathcal{U}_{\Delta} p+\left(\delta_{T} \tilde{\mathcal{Q}}_{0}\right)^{*} \Psi=\tilde{\mathcal{Q}}_{0}^{*}\left(\xi+\left(\mathcal{A}^{*}-\tilde{\mathcal{A}}^{*}\right) p\right)+\left(\delta_{T} \tilde{\mathcal{Q}}_{0}\right)^{*} \Psi
$$

For this $p$, set

$$
\chi_{i} \triangleq \tilde{\mathcal{Q}}_{i}^{*}\left(\xi+\left(\mathcal{A}^{*}-\tilde{\mathcal{A}}^{*}\right) p\right)+\left(\delta_{T} \tilde{\mathcal{Q}}_{i}\right)^{*} \Psi
$$

Let us use Lemma 5.3 now. By this lemma and and by the definitions for the corresponding operators, $\left(p, \chi_{1}, \ldots, \chi_{N}\right) \in Y^{1} \times\left(X^{0}\right)^{N}$ satisfies (5.6). By (5.19) and (5.17), it follows that

$$
p=\tilde{\mathcal{Q}}_{0}^{*}\left(I+\mathcal{U}^{*}\right)^{-1} \xi+\left(I+\mathcal{U}_{\Delta}\right)^{-1}\left(\delta_{T} \tilde{\mathcal{Q}}_{0}\right)^{*} \Psi=\mathcal{Q}_{0}^{*} \xi+\left(\delta_{T} \mathcal{Q}_{0}\right)^{*} \Psi
$$

We have used here that $\left(\delta_{T} \mathcal{Q}_{0}\right)^{*}=\mathcal{Q}_{0}^{*} \delta_{T}^{*}$, where $\mathcal{Q}_{0}^{*}:\left(\mathcal{C}^{0}\right)^{*} \rightarrow X^{1}$ is the linear continuous operator that is adjoint to the linear continuous operator $\mathcal{Q}_{0}: X^{-1} \rightarrow \mathcal{C}^{0}$, and $\delta_{T}^{*}: Z_{T}^{0} \rightarrow\left(\mathcal{C}^{0}\right)^{*}$ is the linear continuous operator that is adjoint to the linear continuous operator $\delta_{T}^{*}: \mathcal{C}^{0} \rightarrow Z_{T}^{0}$. Here $\left(\mathcal{C}^{0}\right)^{*}$ is the space that is dual for $\mathcal{C}^{0}$.

Further, $\chi_{i}$ satisfies

$$
\begin{aligned}
\chi_{i} & =\tilde{\mathcal{Q}}_{i}^{*}\left(\xi+\left(\mathcal{A}^{*}-\tilde{\mathcal{A}}^{*}\right)\left[\mathcal{Q}_{0}^{*} \xi+\left(\delta_{T} \mathcal{Q}_{0}\right)^{*} \Psi\right]\right)+\left(\delta_{T} \tilde{\mathcal{Q}}_{i}\right)^{*} \Psi \\
& =\tilde{\mathcal{Q}}_{i}^{*}\left(\xi+\left(\mathcal{A}^{*}-\tilde{\mathcal{A}}^{*}\right) \tilde{\mathcal{Q}}_{0}^{*}\left(I+\mathcal{U}^{*}\right)^{-1} \xi\right)+\tilde{\mathcal{Q}}_{i}^{*}\left(\mathcal{A}^{*}-\tilde{\mathcal{A}}^{*}\right)\left(\delta_{T} \mathcal{Q}_{0}\right)^{*} \Psi+\left(\delta_{T} \tilde{\mathcal{Q}}_{i}\right)^{*} \Psi \\
& =\tilde{\mathcal{Q}}_{i}^{*}\left(I-\mathcal{U}^{*}\left(I+\mathcal{U}^{*}\right)^{-1}\right) \xi+\tilde{\mathcal{Q}}_{i}^{*}\left(I-\mathcal{U}^{*}\left(I+\mathcal{U}^{*}\right)^{-1}\right) \delta_{T}^{*} \Psi \\
& =\tilde{\mathcal{Q}}_{i}^{*}\left(I+\mathcal{U}^{*}\right)^{-1} \xi+\tilde{\mathcal{Q}}_{i}^{*}\left(I+\mathcal{U}^{*}\right)^{-1} \delta_{T}^{*} \Psi \\
& =\mathcal{Q}_{i}^{*} \xi+\left(\delta_{T} \mathcal{Q}_{i}\right)^{*} \Psi .
\end{aligned}
$$


We have used (5.17) for the last equality. In addition, we have used again that $\left(\delta_{T} \mathcal{Q}_{i}\right)^{*}=\mathcal{Q}_{i}^{*} \delta_{T}^{*}$, where $\mathcal{Q}_{i}^{*}:\left(\mathcal{C}^{0}\right)^{*} \rightarrow X^{0}$ is the linear continuous operator that is adjoint to the linear continuous operator $\mathcal{Q}_{0}: X^{0} \rightarrow \mathcal{C}^{0}$, and $\delta_{T}^{*}: Z_{T}^{0} \rightarrow\left(\mathcal{C}^{0}\right)^{*}$ is the linear continuous operator that is adjoint to the linear continuous operator $\delta_{T}: \mathcal{C}^{0} \rightarrow Z_{T}^{0}$.

Similarly,

$$
\begin{aligned}
p(\cdot, 0) & \left.=\tilde{K}^{*}\left(\xi+\left(\mathcal{A}^{*}-\tilde{\mathcal{A}}^{*}\right) p\right]\right)+\left(\delta_{T} \tilde{K}\right)^{*} \Psi \\
& =\tilde{K}^{*}\left(\xi+\left(\mathcal{A}^{*}-\tilde{\mathcal{A}}^{*}\right)\left[\mathcal{Q}_{0}^{*} \xi+\left(\delta_{T} \mathcal{Q}_{0}\right)^{*} \Psi\right]\right)+\left(\delta_{T} \tilde{K}\right)^{*} \Psi \\
& =\tilde{K}^{*}\left(\xi+\left(\mathcal{A}^{*}-\tilde{\mathcal{A}}^{*}\right) \tilde{\mathcal{Q}}_{0}^{*}\left(I+\mathcal{U}^{*}\right)^{-1} \xi\right)+\tilde{K}^{*}\left(\mathcal{A}^{*}-\tilde{\mathcal{A}}^{*}\right)\left(\delta_{T} \mathcal{Q}_{0}\right)^{*} \Psi+\left(\delta_{T} \tilde{K}\right)^{*} \Psi \\
& =\tilde{K}^{*}\left(I-\mathcal{U}^{*}\left(I+\mathcal{U}^{*}\right)^{-1}\right) \xi+\tilde{K}_{i}^{*}\left(I-\mathcal{U}^{*}\left(I+\mathcal{U}^{*}\right)^{-1}\right) \delta_{T}^{*} \Psi \\
& =\tilde{K}^{*}\left(I+\mathcal{U}^{*}\right)^{-1} \xi+\tilde{K}^{*}\left(I+\mathcal{U}^{*}\right)^{-1} \delta_{T}^{*} \Psi \\
& =K^{*} \xi+\left(\delta_{T} K\right)^{*} \Psi .
\end{aligned}
$$

This completes the proof of Lemma 5.5 .

Remark 5.6 Typically, we use the notations such as $\left(\delta_{T} L\right)^{*}: Z_{T}^{0} \rightarrow X^{0}$ when the entire adjoint operator $\delta_{T} L$ has some sense, so we don't need to take into account properties of the operator $\delta_{T}^{*}$. We use these superposition-type notations for these operators because it reduces the number of symbols for different operators. The only exception is the proof of Lemma 5.5 above, when we consider operators $\left(\delta_{T} \mathcal{Q}_{i}\right)^{*}: Z_{T}^{0} \rightarrow X^{0}$ as the superposition of the operators $\mathcal{Q}_{i}^{*}:\left(\mathcal{C}^{0}\right)^{*} \rightarrow X^{0}$ and $\delta_{T}^{*}: Z_{T}^{0} \rightarrow\left(\mathcal{C}^{0}\right)^{*}$.

\subsection{Proof of Theorem 4.2 for the general case}

Now we are able to prove Theorem 4.2. First, Lemma 5.2 implies that there exists $\zeta \in X^{-1}$ such that $\zeta=P^{*}\left(\xi+\delta_{T}^{*} \Psi+\zeta\right)$, i.e., $\zeta=\left(I-P^{*}\right)^{-1} P^{*}\left(\xi+\delta_{T}^{*} \Psi\right)$. Since the operators $P^{*}:\left(\mathcal{C}^{0}\right)^{*} \rightarrow X^{-1}$ and $\delta_{T}^{*}: Z_{T}^{0} \rightarrow\left(\mathcal{C}^{0}\right)^{*}$ are continuous, we have that $\zeta \in X^{-1}$. Set

$$
p \triangleq \mathcal{Q}_{0}^{*}(\xi+\zeta)+\left(\delta_{T} \mathcal{Q}_{0}\right)^{*} \Psi, \quad \chi_{i} \triangleq \mathcal{Q}_{i}^{*}(\xi+\zeta)+\mathcal{Q}_{i}^{*} \delta_{T}^{*} \Psi, \quad \chi \triangleq\left(\chi_{1}, \ldots, \chi_{N}\right)
$$

Clearly, $(p, \chi) \in Y^{1} \times\left(X^{0}\right)^{N}$. Let us show that the pair $(p, \chi)$ is a solution of problem (4.1). By the definition of the operator $\mathcal{Q}_{i}^{*}$ and by Lemma 5.5, it suffices to show that $\zeta=\sum_{i=1}^{N} B_{i}^{*} \chi_{i}$.

Let $\bar{\zeta} \triangleq \sum_{i=1}^{N} B_{i}^{*} \chi_{i}$. The equations for $\chi_{i}$ are such that

$$
\bar{\zeta}=\sum_{i=1}^{N} B_{i}^{*} \chi_{i}=\sum_{i=1}^{N} B_{i}^{*} \mathcal{Q}_{i}^{*}(\xi+\zeta)+\sum_{i=1}^{N} B_{i}^{*} \mathcal{Q}_{i}^{*} \delta_{T}^{*} \Psi=P^{*}\left(\xi+\delta_{T}^{*} \Psi+\zeta\right) .
$$


The definition of $\zeta$ implies that $\bar{\zeta}=\zeta$. Therefore, the pair $(p, \chi)$ is a solution of problem (4.1). Farther, we have that

$$
\begin{aligned}
p & =\mathcal{Q}_{0}^{*}(\xi+\zeta)+\left(\delta_{T} \mathcal{Q}_{0}\right)^{*} \Psi=\mathcal{Q}_{0}^{*}\left(\xi+\left(I-P^{*}\right)^{-1} P^{*}\left(\xi+\delta_{T}^{*} \Psi\right)\right)+\left(\delta_{T} \mathcal{Q}_{0}\right)^{*} \Psi \\
& =\mathcal{Q}_{0}^{*}\left(I+\left(I-P^{*}\right)^{-1} P^{*}\right)\left[\xi+\left(\delta_{T} \mathcal{Q}_{0}\right)^{*} \Psi\right]=\mathcal{Q}_{0}^{*}\left(I-P^{*}\right)^{-1}\left[\xi+\left(\delta_{T} \mathcal{Q}_{0}\right)^{*} \Psi\right]
\end{aligned}
$$

(we treat $\delta_{T}$ similarly to the proof of Lemma 5.5). By Lemma 5.2, it follows that $p=L^{*} \xi+$ $\left(\delta_{T} L\right)^{*} \Psi$. Similarly,

$$
\begin{aligned}
& \chi_{i}=\mathcal{Q}_{i}^{*}(\xi+\zeta)+\left(\delta_{T} \mathcal{Q}_{i}\right)^{*} \Psi=\mathcal{Q}_{i}^{*}\left(\xi+\left(I-P^{*}\right)^{-1} P^{*}\left(\xi+\delta_{T}^{*} \Psi\right)\right)+\left(\delta_{T} \mathcal{Q}_{i}\right)^{*} \Psi \\
& =\mathcal{Q}_{i}^{*}\left(I+\left(I-P^{*}\right)^{-1} P^{*}\right)\left[\xi+\left(\delta_{T} \mathcal{Q}_{i}\right)^{*} \Psi\right]=\mathcal{Q}_{i}^{*}\left(I-P^{*}\right)^{-1}\left[\xi+\left(\delta_{T} \mathcal{Q}_{i}\right)^{*} \Psi\right] \\
& =\mathcal{M}_{i}^{*} \xi+\left(\delta_{T} \mathcal{M}_{i}\right)^{*} \Psi
\end{aligned}
$$

and

$$
\begin{aligned}
& p(\cdot, 0)=K^{*}(\xi+\zeta)+\left(\delta_{T} K\right)^{*} \Psi=K^{*}\left(\xi+\left(I-P^{*}\right)^{-1} P^{*}\left(\xi+\delta_{T}^{*} \Psi\right)\right)+\left(\delta_{T} K\right)^{*} \Psi \\
& =K^{*}\left(I+\left(I-P^{*}\right)^{-1} P^{*}\right)\left[\xi+\left(\delta_{T} \mathcal{Q}_{0}\right)^{*} \Psi\right]=K^{*}\left(I-P^{*}\right)^{-1}\left[\xi+\left(\delta_{T} \mathcal{Q}_{0}\right)^{*} \Psi\right] \\
& =K^{*} \xi+\left(\delta_{T} K\right)^{*} \Psi .
\end{aligned}
$$

This completes the proof of Theorem 4.2,

\section{Semi-group property for backward equations}

It is known that the dynamic of forward parabolic Ito equation has semi-group property (causality): if $u=L \varphi+\mathcal{L}_{0} \Phi$, where $\varphi \in X^{-1}, \Phi \in Z_{0}^{0}$, then

$$
\left.u\right|_{t \in[\theta, s]}=\left.\left(L \varphi+\mathcal{L}_{0} \Phi\right)\right|_{t \in[\theta, s]}=L(\theta, s) \varphi+\mathcal{L}_{\theta}(\theta, s) u(\cdot, \theta) .
$$

We establish a similar property for the backward equations (anti-causality). This property involves the diffusion term that is a part of the solution.

Theorem 6.1 (Semi-group property for backward equations). Let $0 \leq \theta<s<T$, and let $p=L^{*} \xi, \chi_{i}=\mathcal{M}_{i} \xi$, where $\xi \in X^{-1}$ and $\Psi \in Z_{T}^{0}$. Then

$$
\begin{aligned}
& \left.p\right|_{t \in[\theta, s]}=\left.L(\theta, s)^{*} \xi\right|_{t \in[\theta, s]}+\left(\delta_{s} L(\theta, s)\right)^{*} p(\cdot, s), \\
& p(\cdot, \theta)=\left(\delta_{s} \mathcal{L}_{\theta}(\theta, s)\right)^{*} p(\cdot, s)+\mathcal{L}_{\theta}(\theta, s)^{*} \xi, \\
& \left.\chi_{k}\right|_{t \in[\theta, s]}=\left.\mathcal{M}_{k}(\theta, s)^{*} \xi\right|_{t \in[\theta, s]}+\left(\delta_{s} \mathcal{M}_{i}(\theta, s)\right)^{*} p(\cdot, s), \quad k=1, \ldots, N .
\end{aligned}
$$


Proof of Theorem 6.1. Let $u=L(\theta, s) \varphi+\mathcal{L}_{\theta}(\theta, s) \Phi$, where $\varphi \in X^{0}(\theta, s)$ and $\Phi \in Z_{s}^{1}$ are arbitrary. We have that

$$
\begin{aligned}
& (p(\cdot, s), u(\cdot, s))_{Z_{T}^{0}}-(p(\cdot, \theta), \Phi)_{Z_{T}^{0}} \\
& =\left(-\mathcal{A}^{*} p-\xi-\sum_{i=1}^{N} B_{i}^{*} \chi_{i}, u\right)_{X^{0}(\theta, s)}+(p, \mathcal{A} u+\varphi)_{X^{0}(\theta, s)}+\sum_{i=1}^{N}\left(\chi_{i} B_{i} u\right)_{X^{0}(\theta, s)} .
\end{aligned}
$$

Hence

$$
(p(\cdot, s), u(\cdot, s))_{Z_{T}^{0}}-(p(\cdot, \theta), \Phi)_{Z_{T}^{0}}=-(\xi, u)_{\bar{X}^{0}(\theta, s)}+(p, \varphi)_{X^{0}(\theta, s)}
$$

i.e.,

$$
\begin{aligned}
& (p, \varphi)_{X^{0}(\theta, s)}+(p(\cdot, \theta), \Phi)_{Z_{T}^{0}}=(p(\cdot, s), u(\cdot, s))_{Z_{T}^{0}}+(\xi, u)_{X^{0}(\theta, s)} \\
& =\left(p(\cdot, s), \delta_{s}\left[L(\theta, s) \varphi+\mathcal{L}_{\theta}(\theta, s) \Phi\right]\right)_{Z_{T}^{0}}+\left(\xi, L(\theta, s) \varphi+\mathcal{L}_{\theta}(\theta, s) \Phi\right)_{X^{0}(\theta, s)}
\end{aligned}
$$

Hence

$$
\begin{array}{r}
(p, \varphi)_{\bar{X}^{0}(\theta, s)}+(p(\cdot, \theta), \Phi)_{Z_{T}^{0}}=\left(\left(\delta_{s} L(\theta, s)\right)^{*} p(\cdot, s), \varphi\right)_{X^{0}(\theta, s)}+\left(L(\theta, s)^{*} \xi, \varphi\right)_{X^{0}(\theta, s)} \\
+\left(\left(\delta_{s} \mathcal{L}_{\theta}(\theta, s)\right)^{*} p(\cdot, s), \Phi\right)_{Z_{T}^{0}}+\left(\mathcal{L}_{\theta}^{*} \xi, \Phi\right)_{Z_{T}^{0}} \\
=\left(\left(\delta_{s} L(\theta, s)\right)^{*} p(\cdot, s)+L(\theta, s)^{*} \xi, \varphi\right)_{X^{0}(\theta, s)}+\left(\left(\delta_{s} \mathcal{L}_{\theta}(\theta, s)\right)^{*} p(\cdot, s)+\mathcal{L}_{\theta}(\theta, s)^{*} \xi, \Phi\right)_{Z_{T}^{0}} .
\end{array}
$$

Take $\Phi=0$, then desired equation (6.2) for $\left.p\right|_{[\theta, s]}$ follows. Take $\varphi=0$, then desired equation (6.3) for $p(\cdot, \theta)$ follows.

Similarly, let $u=\mathcal{M}_{k}(\theta, s) h$, where $h \in X^{1}(\theta, s)$ and $k \in\{1, \ldots, N\}$. We have that

$$
\begin{aligned}
& (p(\cdot, s), u(\cdot, s))_{Z_{T}^{0}}=(p(\cdot, s), u(\cdot, s))_{Z_{T}^{0}}-\left(p(\cdot, \theta), u(\cdot, \theta)_{Z_{T}^{0}}\right. \\
& =\left(-\mathcal{A}^{*} p-\xi-\sum_{i=1}^{N} B_{i}^{*} \chi_{i}, u\right)_{X^{0}(\theta, s)}+(p, \mathcal{A} u)_{X^{0}(\theta, s)}+\sum_{i=1}^{N}\left(\chi_{i} B_{i} u\right)_{X^{0}(\theta, s)}+\left(\chi_{k}, h\right)_{X^{0}(\theta, s)} .
\end{aligned}
$$

Hence $(p(\cdot, s), u(\cdot, s))_{Z_{T}^{0}}=-(\xi, u)_{\bar{X}^{0}(\theta, s)}+\left(\chi_{k}, h\right)_{X^{0}(\theta, s)}$, i.e.,

$$
\begin{aligned}
\left(\chi_{k}, h\right)_{X^{0}(\theta, s)} & =(p(\cdot, s), u(\cdot, s))_{Z_{T}^{0}}+(\xi, u)_{X^{0}(\theta, s)} \\
& =\left(p(\cdot, s), \delta_{s} \mathcal{M}_{k}(\theta, s) h\right)_{Z_{T}^{0}}+\left(\xi, \mathcal{M}_{k}(\theta, s) h\right)_{X^{0}(\theta, s)} .
\end{aligned}
$$

Hence $\left(\chi_{k}, h\right)_{X^{0}(\theta, s)}=\left(\left(\delta_{s} \mathcal{M}_{k}(\theta, s)\right)^{*} p(\cdot, s), h\right)_{Z_{T}^{0}}+\left(\mathcal{M}_{k}(\theta, s)^{*} \xi, h\right)_{X^{0}(\theta, s)}$. Then desired equation (6.4) for $\chi_{k}$ follows.

\section{Acknowledgment}

This work was supported by NSERC grant of Canada 341796-2008 to the author. 


\section{References}

Alós, E., León, J.A., Nualart, D. (1999). Stochastic heat equation with random coefficients Probability Theory and Related Fields 115, 1, 41-94.

Bally, V., Gyongy, I., Pardoux, E. (1994). White noise driven parabolic SPDEs with measurable drift. Journal of Functional Analysis 120, 484 - 510.

Chojnowska-Michalik, A., and Goldys, B. (1965). Existence, uniqueness and invariant measures for stochastic semilinear equations in Hilbert spaces, Probability Theory and Related Fields, 102, No. 3, 331-356.

Da Prato, G., and Tubaro, L. (1996). Fully nonlinear stochastic partial differential equations, SIAM Journal on Mathematical Analysis 27, No. 1, 40-55.

Dokuchaev, N.G. (1992). Boundary value problems for functionals of Ito processes, Theory of Probability and its Applications 36 , 459-476.

Dokuchaev, N.G. (1995). Probability distributions of Ito's processes: estimations for density functions and for conditional expectations of integral functionals. Theory of Probability and its Applications 39 (4), 662-670.

Dokuchaev, N.G. (2003). Nonlinear parabolic Ito's equations and duality approach, Theory of Probability and its Applications 48 (1), 45-62.

Dokuchaev, N.G. (2005). Parabolic Ito equations and second fundamental inequality. Stochastics 77, iss. 4., 349-370.

Gyöngy, I. (1998). Existence and uniqueness results for semilinear stochastic partial differential equations. Stochastic Processes and their Applications 73 (2), 271-299.

Kim, Kyeong-Hun (2004). On stochastic partial di!erential equations with variable coefficients in $C^{1}$-domains. Stochastic Processes and their Applications 112, 261-283.

Krylov, N. V. (1999). An analytic approach to SPDEs. Stochastic partial differential equations: six perspectives, 185-242, Math. Surveys Monogr., 64, Amer. Math. Soc., Providence, RI.

Ladyzhenskaia, O.A. (1985). The Boundary Value Problems of Mathematical Physics. New York: Springer-Verlag.

Maslowski, B. (1995). Stability of semilinear equations with boundary and pointwise noise, Ann. Scuola Norm. Sup. Pisa Cl. Sci. (4), 22, No. 1, 55-93.

Pardoux, E., S. Peng, S. (1990). Adapted solution of a backward stochastic differential equation. System \& Control Letters 14, 55-61. 
Pardoux, E. (1993). Stochastic partial differential equations, a review, Bull. Sc. Math. 117, $29-47$.

Rozovskii, B.L. (1990). Stochastic Evolution Systems; Linear Theory and Applications to Non-Linear Filtering. Kluwer Academic Publishers. Dordrecht-Boston-London.

Walsh, J.B. (1986). An introduction to stochastic partial differential equations, Ecole d'Eté de Prob. de St. Flour XIV, 1984, Lect. Notes in Math 1180, Springer Verlag.

Yong, J., and Zhou, X.Y. (1999). Stochastic controls: Hamiltonian systems and HJB equations. New York: Springer-Verlag.

Yosida, K. (1965). Functional Analysis. Springer-Verlag. Berlin, Gottingen, Heidelberg.

Zhou, X.Y. (1992). A duality analysis on stochastic partial differential equations, Journal of Functional Analysis 103, No. 2, 275-293. 